\title{
SUBAVERAGING ESTIMATES FOR CR FUNCTIONS
}

\author{
A. BOGGESS AND R. DWILEWICZ
}

(Communicated by Irwin Kra)

\begin{abstract}
We give conditions on a CR submanifold $M$ in $\mathbf{C}^{n}$ and a compact submanifold $N \subset M$ such that the average value on $N$ of a CR function on $M$ can be estimated uniformly by the $L^{1}$-norm of the CR function on a neighborhood of $N$ in $M$. The conditions involve the Levi form of $M$ and the transversality of $N$ to the holomorphic tangent bundle of $M$.
\end{abstract}

1. Introduction and statement of results. If $f$ is a holomorphic function on a domain $\tilde{\Omega}$ in $\mathbf{C}^{n}$ and if $\Omega_{1} \Subset \Omega \Subset \tilde{\Omega}$ then it is well known (see $[\mathbf{H}$, Theorem 2.2.3]) that there is a constant $K$ which depends only on $\Omega_{1}$ and $\Omega$ with

$$
|f(z)| \leq K\|f\|_{\mathscr{L}^{1}(\Omega)}
$$

for all $z \in \Omega_{1}$. If $N$ is a closed submanifold of $\tilde{\Omega}$, then one can easily integrate (1.1) over $N$ to obtain the following subaveraging estimate

$$
\left|\int_{N} f d \sigma_{N}\right| \leq K\|f\|_{\mathscr{L}^{1}(\Omega)}
$$

where $\Omega \subset \tilde{\Omega}$ is an open set which contains $N$ and $K$ depends only on $N, \Omega$ and $\tilde{\Omega}$. Here $d \sigma_{N}$ is surface measure on $N$.

Now an estimate analogous to (1.1) for CR functions on a CR submanifold $M$ generally fails. For example on a strictly pseudoconvex real hypersurface, the existence of peak functions implies that (1.1) fails. It is then natural to investigate whether or not the subaveraging estimate (1.2) holds for CR functions. This paper carries out this investigation. Our result (Theorem 1.1 below) in particular implies that if $M$ is a real hypersurface whose Levi form does not vanish at each point of $N \subset M$, then the subaveraging estimate holds provided $N$ is always transverse to the holomorphic tangent bundle to $M$. Thus, there are plenty of cases (e.g. $M$ strictly pseudoconvex) in which the subaveraging estimate (1.2) holds but (1.1) fails. Counterexamples (to (1.2)) are provided in $\S 3$ in the case when $N$ is not holomorphically transverse.

Our theorem handles the case when $\operatorname{codim}_{\mathbf{R}} M \geq 1$ and therefore we need some additional notation before we can state our result.

Let $M$ be a smooth generic $\mathrm{CR}$ submanifold in $\mathrm{C}^{n}$ with $\operatorname{codim}_{\mathbf{R}} M \geq 1$. Let $H(M)$ be the holomorphic tangent bundle of $M$ with fibre $H_{p}(M), p \in M$. We also let $N_{p}(M)$ be the space of vectors which are orthogonal (under the Euclidean metric in $\left.\mathbf{R}^{2 n}\right)$ to the real tangent space of $M$ at $p\left(T_{p}(M)\right)$ and we let

$$
\pi_{p}: T_{p}\left(\mathbf{R}^{2 n}\right) \rightarrow N_{p}(M)
$$

Received by the editors February 19, 1987 and, in revised form, June 5, 1987.

1980 Mathematics Subject Classification (1985 Revision). Primary 32A40, 32F25.

(C) 1988 American Mathematical Society $0002-9939 / 88 \$ 1.00+\$ .25$ per page 
be the orthogonal projection. The first Levi form $\mathscr{L}_{p}: H_{p}(M) \rightarrow N_{p}(M)$ is defined by

$$
\mathscr{L}_{p}\left(X_{p}\right)=\pi_{p} J[X, J X]_{p}, \quad X_{p} \in H_{p}(M)
$$

where $X \in H(M)$ is any vector field extension of $X_{p}$ and $J$ is the usual complex structure map on $\mathbf{R}^{2 n} \simeq \mathbf{C}^{n}$. Note that $\pi_{p} J v \in N_{p}(M)$ for $v \in T_{p}(M)$ and $\pi_{p}(J v) \neq 0$ if and only if $v \notin H_{p}(M)$. We let $\Gamma_{p}$ be the convex hull of the image of $\mathscr{L}_{p}$ in $N_{p}(M)$. The cone $\Gamma_{p}$ essentially determines the size of the normal cross section of the open set to which CR functions on $M$ extend as holomorphic functions (see $[\mathbf{B P}])$.

Now we are in a position to formulate our main theorem and some of its consequences.

1.1. THEOREM. Let $N$ be a compact submanifold of $M$ of $\operatorname{dim}_{\mathbf{R}} N \geq 1$, without boundary. We assume the following on $N$ and $M$ :

For each $p \in N \subset M$, the interior of $\Gamma_{p}$ (with respect to the topology on $\left.N_{p}(M)\right)$ is nonempty.

Then for a given neighborhood $\omega$ of $N$ in $M$ there is a constant $C=C(N, \omega)$ such that for each $C R$ function $f$ on $\omega$

$$
\left|\int_{N} f d \sigma_{N}\right| \leq C \int_{\omega}|f| d \sigma_{M}
$$

where $d \sigma_{N}, d \sigma_{M}$ are surface measures on $N$ and $M$, respectively.

When $M$ is a real hypersurface with nonvanishing Levi form, requirement (1.3a) is automatically satisfied because $N_{p}(M)$ is just a one-dimensional line which is normal to $M$. If $N \subset M$ is a compact submanifold of $M$ without boundary with $T_{p}(N)$ transverse to $H_{p}(M)$ (i.e. $\left.T_{p}(N) \not \subset H_{p}(M)\right)$ then requirement $(1.3 \mathrm{~b})$ is satisfied. Thus we have the following important corollary.

1.2. COROLlARY. Let $M$ be a real hypersurface of $\mathbf{C}^{n}$ and $N \subset M$ a compact submanifold of $M$ without boundary, $\operatorname{dim}_{\mathbf{R}} N \geq 1$, such that $\mathscr{L}_{p}(M) \not \equiv 0$ for each $p \in N$. If $T_{p}(N) \not \subset H_{p}(M)$ for each $p \in N$, then the conclusion of Theorem 1.1 holds.

Another special case of Theorem 1.1 is the following.

1.3. COROllary. Let $M$ be a $C R$ submanifold of $\mathbf{C}^{n}$ with $\operatorname{codim}_{\mathbf{R}} M=k \geq 1$. Let $N \subset M$ be a submanifold of $M$ with $\operatorname{dim}_{\mathbf{R}} N=k$ and such that (1.3a) holds. If $T_{p}(N) \cap H_{p}(M)=\varnothing$ for each $p \in N$ then the conclusion of Theorem 1.1 holds.

To prove the above corollary, we note that if $T_{p}(N) \cap H_{p}(M)=\varnothing$, then $\pi_{p} J T_{p}(N)$ $=N_{p}(M)$ and therefore $(1.3 \mathrm{~b})$ is satisfied.

If $M$ is a convex hypersurface and $N \subset M$ is a one-dimensional curve which is the boundary of an analytic disc $D$ which intersects $M$ transversally, then estimate (1.3c) follows easily. One merely thinks of $D$ as part of a family of analytic discs with boundaries in $M$ and then by extending the given CR function to a holomorphic function to the inside of $M$, estimate (1.3c) follows from the subaveraging 
properties of holomorphic functions (see [L and $\mathbf{W}]$ ). Therefore our theorem is more meaningful in situations where $N$ is not the boundary of some analytic object.

The next section is devoted to the proof of our theorem and the final section contains some counterexamples to estimate $(1.3 \mathrm{c})$ in the case when $(1.3 \mathrm{~b})$ is violated.

2. Proof of the Main Theorem. The outline of the proof is as follows. We realize $N$ as the boundary of a manifoldf $\tilde{N} \subset \mathbf{C}^{n}$ whose coordinate functions satisfy a Cauchy-Riemann equation to infinite order at $N$. The transversality condition (1.3b) will ensure that $\tilde{N}$ will be transverse to $M$. We then use Henkin's integral kernels to represent the given CR function and with the help of Stoke's theorem, we transfer the integration over $N$ which appears on the left side of (1.3c) to an integration over $\tilde{N}$. The transversality of $\tilde{N}$ to $M$ allows us to estimate Henkin's kernels on $\tilde{N}$ and the estimate (1.3c) will follow.

Henkin's extension formulas. We fix $N \subset M$ and the open set $\omega$ in $M$ which contains $N$ as given in the hypothesis of Theorem 1.1. We let $f$ be a CR function on $\omega$. For each $p \in N$, assumption (1.3a) allows us to extend $f$ as a holomorphic function $F$ defined on an open set $\mathscr{O}_{\rho}$ in $\mathbf{C}^{n}$. The size of the cross section of $\mathscr{O}_{\rho}$ is determined by the cone $\Gamma_{p}$, which by definition is the convex hull of the image of the Levi form $\mathscr{L}_{p}: H_{p}(M) \rightarrow N_{p}(M)$. More precisely, given any smaller cone $\Gamma^{\prime}<\Gamma_{p}$ there is an open set $\omega_{p}$ in $M$ containing $p$ and $\delta>0$ such that

$$
\omega_{p}+\left\{\Gamma^{\prime} \cap B(0, \delta)\right\} \subset \mathscr{O}_{p}
$$

Here, $B(0, \delta)$ represents the ball centered at the origin $(=p)$ in $N_{p}(M)$ and the notation $\Gamma^{\prime}<\Gamma_{p}$ means that $\left\{\Gamma^{\prime} \cap S\right\} \Subset \Gamma_{p} \cap S$ where $S$ is the unit sphere in $N_{p}(M)$.

Using Henkin's integral formulas (see [AH, Theorem 5.2.1]) we can write down an explicit formula for $F$. We let $D(p, r)$ be the set $\{q \in M:|q-p|<r\}$. There is a choice of $r_{p}>0, \delta>0$ and the set $\omega_{p}$ can be shrunk if ncessary so that if $r_{p} / 2 \leq r \leq r_{p}$ then

$$
F(w)=\int_{\zeta \in D(p, r)} K_{1}(\zeta, w) f(\varsigma)+\int_{\zeta \in \partial(D(p, r))} K_{2}(\zeta, w) f(\zeta)
$$

for $w \in \omega_{p}+\left\{\Gamma^{\prime} \cap B(0, \delta)\right\}$. Here $K_{1}$ and $K_{2}$ are explicit kernels and all we need to know about these kernels is that they satisfy the following estimates:

$$
\left|K_{j}(\zeta, w)\right| \leq C /|\zeta-w|^{2 n}, \quad\left|\nabla_{w} K_{j}(\zeta, w)\right| \leq C /|\zeta-w|^{2 n+2}, \quad j=1,2,
$$

which holds for $\varsigma \in \overline{D(p, r)}, w \in \omega_{p}+\left\{\Gamma^{\prime} \cap B(0, \delta)\right\}$ and where the constant $C$ is independent of $\zeta, w$ and $r$ if say $r_{p} / 2 \leq r \leq r_{p}$. The constant may depend on $\omega_{p}$, $\Gamma^{\prime}$ and $r_{p}$.

Extension of the submanifold $N$. Assumption (1.3a) allows us to say that for each $p \in M$ there is a nonzero vector $v_{p} \in T_{p}(N)$ so that the nonzero vector $\pi_{p} J\left(v_{p}\right)$ lies in the interior of $\Gamma_{p}$. Now we can locally parameterize $N$ by a $C^{\infty} \operatorname{map} G: \mathbf{R}^{k} \rightarrow N$ with $G(0)=p$. We give coordinates $(x, u)$ to $\mathbf{R}^{k}$, with $x \in \mathbf{R}, u \in \mathbf{R}^{k-1}$ chosen so that $G_{*}\left(\partial /\left.\partial x\right|_{0}\right)=v_{p}$, where $G_{*}$ represents the differential of $G$. Since $\pi_{p} J v_{p}$ lies in the interior of $\Gamma_{p}$, clearly we can choose a cone $\Gamma_{p}^{\prime \prime}<\Gamma_{p}$ and an open set $\omega_{p}$ 
containing $p$ such that

$$
\pi_{p} J\left\{G_{*}\left(\left.\frac{\partial}{\partial x}\right|_{(x, u)}\right)\right\} \in \Gamma_{p}^{\prime \prime}
$$

for all $(x, u) \in G^{-1}\left(\omega_{p} \cap N\right)$.

We give to $\mathbf{C} \times \mathbf{R}^{k-1}$ the coordinates $(z, u)$ where $z=x+i y \in \mathbf{C}$ and where $(x, u)$ are the coordinates of $\mathbf{R}^{k}$ chosen above. We extend $G(x, u)$ to $G(z, u)$ in such a way that $(\partial G / \partial \bar{z})(x+i y, u)$ vanishes to infinite order at $y=0$. In particular, $G$ satisfies the Cauchy-Riemann equations in the variable $z$ when $y=\operatorname{Im} z=0$. Therefore in view of $(2.4)$ we have

$$
\pi_{p} \frac{\partial G}{\partial y}(x+i 0, u)=\pi J_{p}\left\{\frac{\partial G}{\partial x}(x+i 0, u)\right\} \in \Gamma_{p}^{\prime \prime}<\Gamma_{p}
$$

for all $(x, u) \in G^{-1}\left(\omega_{p} \cap N\right)$.

Fix a $\delta^{\prime}>0$ (to be chosen later). Define for $0 \leq t<\delta^{\prime}$

$$
\begin{aligned}
\tilde{N}_{t, \delta^{\prime}} & =\left\{G(x+i y, u) ;(x, u) \in G^{-1}\left(\omega_{p} \cap N\right), t \leq y<\delta^{\prime}\right\}, \\
N_{t} & =\left\{G(x+i t, u) ;(x, u) \in G^{-1}\left(\omega_{p} \cap N\right)\right\} .
\end{aligned}
$$

Note that $N_{0}=N \cap \omega_{p}$, and also note that the tangent cone of $\tilde{N}_{0, \delta^{\prime}}$ at $G(x, u)$ is spanned by the tangent space of $N$ at $G(x, u)$ and by the vector $(\partial G / \partial y)(x+i 0, u)$ for each $(x, u) \in G^{-1}\left(\omega_{p} \cap N\right)$. Choose a cone $\Gamma_{p}^{\prime}$ with $\Gamma_{p}^{\prime \prime}<\Gamma_{p}^{\prime}<\Gamma_{p}$. In view of (2.5) and the above discussion, $\delta^{\prime}>0$ can be chosen and $\omega_{p}$ can be shrunk if necessary so that

$$
\tilde{N}_{0, \delta^{\prime}} \subset \omega_{p}+\left\{\Gamma_{p}^{\prime} \cap B(0, \delta)\right\}
$$

where $\delta$ is chosen as in (2.1). Note in particular that (2.6) implies that $\tilde{N}_{0, \delta^{\prime}}$ is transverse to $M$. We choose an open set $\omega_{p} \ni p$ which satisfies the requirement of (2.1), (2.2) and (2.6). We get an open cover $\left\{\omega_{p}, p \in N\right\}$ of $N$. By the compactness of $N$ we can extract a finite number of points $\left.p_{j}\right|_{j=1} ^{l}$ with corresponding open sets $\left.\omega_{p_{j}}\right|_{j=1} ^{l}$ which cover $N$. We chose a partition of unity $\left.\psi_{j}\right|_{j=1} ^{l}$ subordinate to the cover $\left.\omega_{j} \cap N\right|_{j=1} ^{l}$ (so $\psi_{j}$ is only defined on $N \cap \omega_{j}$ ).

We have

$$
\int f d \sigma_{N}=\sum_{j=1}^{l} \int_{N \cap \omega_{p_{j}}} f \psi_{j} d \sigma_{N}=\sum_{j=1}^{l} \int_{N \cap \omega_{p_{j}}} F_{j} \psi_{j} d \sigma_{N}
$$

where $F_{j}$ is the holomorphic extension of $f$ defined on $\omega_{p_{j}}+\left\{\Gamma_{p_{j}}^{\prime} \cap B\left(0, \delta_{p_{j}}\right)\right\}$. We shall estimate each term in the above sum on the right and for simplicity we shall drop the subscript $j$ (so $\omega_{p}=\omega_{p_{j}}, \Gamma_{p_{j}}^{\prime}=\Gamma_{p}^{\prime}, F_{j}=F$ and $\psi_{j}=\psi$ etc.).

Completion of the proof of the theorem. Let $\phi(x, u)$ be the smooth compactly supported function in $G^{-1}\left(\omega_{p} \cap N\right) \subset \mathbf{R}^{k}$ defined by

$$
\phi d x d u=G^{*}\left(\psi d \sigma_{N}\right) \text {. }
$$

We extend $\phi$ to $\phi(z, u)$ on $\mathbf{C} \times \mathbf{R}^{k-1}$ so that $(\partial \phi / \partial \bar{z})(z, u)$ vanishes to infinite order at $y=\operatorname{Im} z=0$. We may also assume that $\phi(x+i y, u)=0$ for $y \geq \delta^{\prime} / 2$ where $\delta^{\prime}$ is the number in the definition of $\tilde{N}_{t, \delta^{\prime}}$. We let

$$
\Phi=G^{-1^{*}}\{\phi(z, u) d z d u\} .
$$


We note that

$$
\int_{N} F \psi d \sigma_{N}=\int_{N_{0}} F \Phi
$$

So we shall obtain an estimate for $\int_{N_{t}} F \Phi$ which is uniform in $t>0$ and then let $t \rightarrow 0$.

Using Stoke's theorem and the fact that $\Phi$ vanishes on $N_{t}$ for $t \geq \delta^{\prime} / 2$, we obtain

$$
\int_{N_{t}} F \Phi=\int_{\tilde{N}_{t, \delta^{\prime}}}(d F \wedge \Phi+F \wedge d \Phi) .
$$

Now $F$ is holomorphic on a neighborhood of $\tilde{N}_{t, \delta^{\prime}}$ because $\tilde{N}_{t, \delta^{\prime}} \subset \tilde{N}_{0, \delta^{\prime}} \subset \omega_{p}+$ $\left\{\Gamma_{p}^{\prime} \cap B(0, \delta)\right\}$ (see (2.6)). Therefore $d F=\partial F$ in the first integral on the right of (2.7) above. We pull this integral back via $G$ to $\mathbf{C} \times \mathbf{R}^{k-1}$ to obtain

$$
\begin{aligned}
\int_{\tilde{N}_{t, \delta^{\prime}}} \partial F \wedge \Phi & =\sum_{l=1}^{n} \int_{\begin{array}{c}
t \leq y \leq \delta^{\prime} \\
(z=x+i y)
\end{array}} \int_{(x, u) \in \mathbf{R}^{k}} \frac{\partial F}{\partial w_{l}}(G(z, u)) d G_{l} \wedge \phi(z, u) d z \wedge d u \\
& =\sum_{l=1}^{n} \int_{t \leq y \leq \delta^{\prime}} \int_{(x, u) \in \mathbf{R}^{k}} \frac{\partial F}{\partial w_{l}}(G(z, u)) \frac{\partial G_{l}}{\partial \bar{z}}(z, u) \phi(z, u) d \bar{z} \wedge d z \wedge d u .
\end{aligned}
$$

For $q \in \mathbf{C}^{n}$, let $\operatorname{dist}(q)$ be the Euclidean distance from $q$ to $M$. We claim for some $c>0$

$$
\operatorname{dist}(G(x+i y, u)) \geq c|y| \text { for } 0 \leq y<\delta^{\prime} .
$$

To prove this estimate we note that $G(x, u) \in M$ and $(\partial G / \partial y)(x, u)$ is a vector which is transverse to $M$. This is because $\tilde{N}_{0, \delta}$ is transverse to $M$. More precisely, the function $G$ which parameterizes $\tilde{N}_{0, \delta^{\prime}}$ satisfies the Cauchy-Riemann equations at $(x+i 0, u) \in \mathbf{R}^{k}$, i.e. $(\partial G / \partial y)(x+i 0, u)=J((\partial G / \partial x)(x+i 0, u))$ and because the vector $(\partial G / \partial x)(x+i 0, u)$ is an element of $T_{\tilde{p}}(M)$ but not an element of $H_{\tilde{p}}(M)$ where $\tilde{p}=G(x, u)$. Therefore there is a positive constant $c>0$ with

$$
\operatorname{dist}(G(x+i y, u)) \geq c|G(x+i y, u)-G(x, u)| \geq \frac{c}{2}\left|\frac{\partial G}{\partial y}(x, u)\right||y|
$$

and (2.9) is established.

In view of (2.9) and (2.3), we have that for $\varsigma \in M$

$$
\begin{aligned}
\left|\frac{\partial K_{j}}{\partial w_{l}}(G(z, u), \varsigma)\right| & \leq \frac{C}{|G(z, u)-\varsigma|^{2 n+2}} \\
& \leq \frac{C}{\operatorname{dist}(G(x+i y, u))^{2 n+2}} \leq \frac{C}{|y|^{2 n+2}}
\end{aligned}
$$

where $C$ is a uniform constant which is independent of $x, y, u$ and $\zeta$. In addition $\partial G_{l} / \partial \bar{z}$ vanishes to infinite order at $y=0$. Therefore

$$
\left|\frac{\partial G}{\partial \bar{z}}(x+i y, u)\right| \leq C|y|^{2 n+2}
$$

for some uniform constant $C$. By inserting formula (2.2) for $F$ into the right side of $(2.8)$ and then by using estimates $(2.10)$ and $(2.11)$, we obtain

$$
\left|\int_{\tilde{N}_{t, \delta^{\prime}}} \partial F \wedge \Phi\right| \leq C\left[\int_{\zeta \in D(p, r)}|f(\zeta)| d \sigma(\zeta)+\int_{\zeta \in \partial D(p, r)}|f(\zeta)| d \sigma(\zeta)\right]
$$


where the constant $C$ is independent of $f$ and $t$ with $0 \leq t<\delta^{\prime}$ and $r$ with $r_{p} / 2 \leq r \leq r_{p}$. Letting $t \rightarrow 0$ we obtain the same inequality as in (2.12) with $t$ replaced by 0 .

The second integral on the right side of $(2.7)$ is handled similarly. We pull it back via $G$ to obtain

$$
\int_{t \leq y \leq \delta^{\prime}} \int_{(x, u) \in \mathbf{R}^{k}} F(G(z, u)) \frac{\partial \phi}{\partial \bar{z}}(z, u) d \bar{z} \wedge d z \wedge d u .
$$

Since $\partial \phi / \partial \bar{z}$ vanishes to infinite order, one can repeat the above arguments and estimate this term by the right side of (2.12). Thus combining these estimates for both terms on the right side of (2.7) (with $t=0$ ) we obtain

$$
\left|\int_{N} \psi f d \sigma_{N}\right| \leq C\left[\int_{\zeta \in D(p, r)}|f(\varsigma)| d \sigma(\varsigma)+\int_{\zeta \in \partial D(p, r)}|f(\varsigma)| d \sigma(\varsigma)\right]
$$

where the constant $C$ is independent of $f$ and $r, r_{p} / 2 \leq r \leq r_{p}$. We now integrate the above estimate as $r$ goes from $r_{p} / 2$ to $r_{p}$ to obtain

$$
\left|\int_{N} \psi f d \sigma_{N}\right| \leq C\left[\int_{\varsigma \in D\left(p, r_{p}\right)}|f(\zeta)| d \sigma(\varsigma)\right] .
$$

Since $\psi\left(=\psi_{j}\right)$ is an arbitrarily chosen element of the partition of unity, we may sum over $j, 1 \leq j \leq l$ and obtain the estimate

$$
\left|\int_{N} f d \sigma_{N}\right| \leq C \int_{\omega}|f| d \sigma_{M}
$$

as desired.

3. Examples. In this section we give two counterexamples to estimate (1.3c) if the assumption $(1.3 \mathrm{~b})$ is violated.

The first example concerns the case where $M$ is a hypersurface and $N$ is a curve on $M$ which is tangent at some point $p$ to the space $H_{p}(M)$. Moreover we shall see how the constant $C$ in (1.3c) depends on transversality of $N$ with respect to the complex tangent space of $M$. This all is done in $\mathbf{C}^{2}$ because the situation in $\mathbf{C}^{n}$ is analogous.

The second example shows that if the codimension of $M$ is higher than 1 , the assumption of transversality of $N$ with respect to the complex tangent space of $M$ is not enough. In this case, the constant $C$ in (1.3c) reflects the extent to which the spaces $\pi_{p} J T_{p}(N), p \in N$, lie inside to the convex hull of the image of the Levi form. In the example given, the codimension of $M$ is 2 and $N$ is a curve on $M$, but analogous examples can be easily generated for any dimensions.

EXAMPLE 3.1. Let $M$ be a hypersurface in $\mathbf{C}^{2}$ given by

$$
M=\left\{\left(z_{1}, z_{2}\right) \in \mathbf{C}^{2} ; \operatorname{Re} z_{2}=\left|z_{1}\right|^{2} \text { and }\left(z_{1}, \operatorname{Im} z_{2}\right) \in B\right\}
$$

where

$$
B=\left\{\left(z_{1}, y_{2}\right) \in \mathbf{C} \times \mathbf{R} ;\left|z_{1}\right|<1,\left|y_{2}\right|<1\right\} .
$$

Fix two positive, sufficiently small real numbers $\lambda, \mu$. For any $a \in \mathbf{R}, a \geq 0$, define a closed curve $N^{a}$ passing through $0=(0,0)$, and parameterized by 
$z(t)=\left(z_{1}(t), z_{2}(t)\right), t \in[-1,1]$, where $z(-1)=z(1)$. Moreover assume that the parameterization satisfies two conditions:

$$
\begin{gathered}
z_{1}(t)=t, \quad z_{2}(t)=t^{2}+i a t \quad \text { for }|t| \leq \lambda, \\
\operatorname{Re} z_{2}(t) \geq \mu \quad \text { for } \lambda \leq|t| \leq 1 .
\end{gathered}
$$

Notice that if $a=0$ then $N^{0}$ is tangent to $H_{0}(M)$ and if $a>0$ then $N^{a}$ is transverse to this space.

For any positive $\varepsilon$ define the smooth $\mathrm{CR}$ function

$$
f_{\varepsilon}(z)=1 /\left(z_{2}+\varepsilon\right), \quad z \in M
$$

Consider both integrals which appear in inequality (1.3c). By $C_{1}, C_{2}, \ldots$ denote some positive constants.

Upper estimate of the integral $\int_{M}\left|f_{\varepsilon}\right| d \sigma_{M}$.

$$
\int_{M}\left|f_{\varepsilon}\right| d \sigma_{M} \leq C_{1} \int_{B}\left[\left(\left|z_{1}\right|^{2}+\varepsilon\right)^{2}+y_{2}^{2}\right]^{1 / 2} d x_{1} d y_{1} d y_{2} .
$$

The integral on the right of (3.4) can be estimated for small $\varepsilon$ by

$$
\int_{B}\left(\varepsilon^{2}+y_{2}^{2}\right)^{-1 / 2} d x_{1} d y_{1} d y_{2}=\pi \int_{-1}^{1}\left(\varepsilon^{2}+y_{2}^{2}\right)^{-1 / 2} d y_{2} \leq-C_{2} \ln \varepsilon
$$

Consequently, (3.4) and (3.5) gives

$$
\int_{M}\left|f_{\varepsilon}\right| d \sigma_{M} \leq-C_{1} C_{2} \ln \varepsilon
$$

Lower estimate of the integral $\left|\int_{N^{0}} f_{\varepsilon} d \sigma_{N}\right|$. Using (3.2) and (3.3) we obtain

$$
\left|\int_{N^{0}} f_{\varepsilon} d \sigma_{N}\right| \geq \int_{-\lambda}^{\lambda} \frac{1}{t^{2}+\varepsilon} d t-C_{3} \geq C_{4} \varepsilon^{-1 / 2}-C_{3} .
$$

If $\varepsilon$ is small, then (3.6) and (3.7) show that (1.3c) cannot be satisfied.

If $a \geq 0$, the following generalization of (3.7) can be proved:

$$
\left|\int_{N^{a}} f_{\varepsilon} d \sigma_{N}\right| \geq C_{5}\left(a^{4}+\varepsilon^{2}\right)^{-1 / 4}
$$

where $C_{5}$ does not depend on $a$ and $\varepsilon$. The last inequality together with (3.6) show how the constant $C$ in (1.3c) depends on $a$ which reflects transversality of $N^{a}$ with respect to $H_{0}(M)$.

EXAMPLE 3.2. Let $M$ be a real submanifold of $\mathrm{C}^{4}$ given by the equations

$$
\operatorname{Re} z_{3}=\left|z_{1}\right|^{2}, \quad \operatorname{Re} z_{4}=\left|z_{2}\right|^{2}
$$

for $z \in B=\left\{z \in \mathbf{C}^{4} ;\left|z_{\alpha}\right|<1,\left|y_{\beta}\right|<1, \alpha, \beta=1,2\right\}$ where $z_{\alpha}=x_{\alpha}+i y_{\alpha}, a=$ $1,2,3,4$. The complex tangent space $H_{0}(M)$ is spanned by $\partial / \partial z_{1}$ and $\partial / \partial z_{2}$. The Levi form $\mathscr{L}_{0}(\varsigma)$ for $\varsigma=\varsigma_{1} \partial / \partial z_{1}+\varsigma_{2} \partial / \partial z_{2} \in H_{0}(M)$ is

$$
\mathscr{L}_{0}(\varsigma)=\varsigma_{1} \bar{\zeta}_{1} \frac{\partial}{\partial x_{3}}+\varsigma_{2} \bar{\zeta}_{2} \frac{\partial}{\partial x_{4}}
$$

and its image covers the first quadrant of the plane spanned by $\partial / \partial x_{3}$ and $\partial / \partial x_{4}$. 
Fix two positive sufficiently small real numbers $\lambda, \mu$. For any $(a, b) \in \mathbf{R}^{2}$ define a closed curve $N^{a, b}$ parameterized by $z(t)=\left(z_{\alpha}(t)\right)_{\alpha=1,2,3,4}, t \in[-1,1]$, where $z(-1)=z(1)$. Moreover assume that the parameterization satisfies the following conditions:

$$
\begin{gathered}
z_{1}(t)=t, \quad z_{2}(t)=t, \quad z_{3}(t)=t^{2}+i a t, \quad z_{4}(t)=t^{2}+i b t \quad \text { for }|t| \leq \lambda, \\
\operatorname{Re} z_{3}(t) \geq \mu, \quad \operatorname{Re} z_{4}(t) \geq \mu \quad \text { for } \lambda \leq|t| \leq 1 .
\end{gathered}
$$

Notice that $N^{0,0}$ is tangent to $H_{0}(M)$ and if $(a, b) \neq(0,0)$ then $N^{a, b}$ is transverse to $H_{0}(M)$. If $a b>0$ then $N^{a, b}$ satisfies (1.3b) in a neighborhood of the origin. If $a b \leq 0$ then $(1.3 \mathrm{~b})$ is not satisfied at the origin.

For any $\varepsilon>0, c \geq 0, d \geq 0,(c, d) \neq(0,0)$ define the smooth CR function

$$
f_{\varepsilon}^{c, d}(z)=\frac{1}{c z_{3}+d z_{4}+\varepsilon}, \quad z \in M .
$$

Similarly as in the previous example, it can be shown that if $a b \leq 0$, say $a \geq 0$ and $b \leq 0$, then there exist some positive constants $C_{1}, C_{2}, C_{3}$ that for small $\varepsilon$,

$$
\int_{M}\left|f_{\varepsilon}^{c, d}\right| d \sigma_{M} \leq-C_{1} \ln \varepsilon, \quad\left|\int_{N^{a, b}} f_{\varepsilon}^{c, d} d \sigma_{N}\right|>C_{2} \varepsilon^{-1 / 2}-C_{3}
$$

for $c=-b, d=a$ if $(a, b) \neq(0,0)$ or any positive $c, d$ if $(a, b)=(0,0)$. Consequently inequality $(1.3 \mathrm{c})$ is not satisfied for small $\varepsilon$.

If $a>0$ and $b>0, a^{2}+b^{2}=1$, i.e. (1.3b) is satisfied in a neighborhood of the origin, the following inequality can be proved:

$$
\inf _{c, d}\left|\int_{N^{a, b}} f_{\varepsilon}^{c, d} d \sigma_{N}\right| \geq C_{4}\left\{[\min (a, b)]^{4}+\varepsilon^{2}\right\}^{-1 / 4}
$$

where the infimum is over nonnegative $c, d$ such that $c^{2}+d^{2}=1$, and the constant $C_{4}$ does not depend on $a, b$ and $\varepsilon$. Notice that $\min (a, b)$ gives the angle between the line $\pi_{0} J T_{0}\left(N^{a, b}\right)$ and the closest side of the complement of the image of the Levi form. The last inequality together with the first inequality (3.8) show how the constant $C$ in (1.3c) depends on the position of the spaces $\pi_{p} J T_{p}(N)$ in the image of the Levi form.

\section{REFERENCES}

[AH] R. A. Airapetyan and G. M. Henkin, Integral representations of differential forms on CRmanifolds and the theory of CR-functions, Uspekhi Mat. Nauk 39 (1984), 39-106; English transl., Russian Math. Surveys 39 (1984), 41-118.

[BP] A. Boggess and J. C. Polking, Holomorphic extension of CR functions, Duke Math. J. 49 (1982), 757-784.

[H] L. Hörmander, An introduction to complex analysis in several variables, North-Holland, 1973.

[L] H. Lewy, On the local character of the solutions of an atypical linear differential equation in three variables and a related theorem for regular functions of two complex variables, Ann. of Math. 64 (1956), 514-522.

[W] R. O. Wells, Jr., On the local holomorphic hull of a real submanifold in several complex variables, Comm. Pure Appl. Math. 19 (1966), 145 165.

Department of Mathematics, Texas a\&M University, College Station, TEXAS 77843

Institute of Mathematics, Warsaw University, 00901 Warsaw, Poland 